\title{
Practical Aspects on Non-profiled Deep-learning Side-channel Attacks against AES Software Implementation with Two Types of Masking Countermeasures including RSM
}

\author{
Kunihiro Kuroda \\ Ritsumeikan university \\ Kusatsu, Shiga, Japan \\ ri0080rr@ed.ritsumei.ac.jp
}

\author{
Yuta Fukuda \\ Ritsumeikan university \\ Kusatsu, Shiga, Japan \\ ri0073pi@ed.ritsumei.ac.jp
}

\author{
Kota Yoshida \\ Ritsumeikan university \\ Kusatsu, Shiga, Japan \\ ri0044ep@ed.ritsumei.ac.jp
}

\author{
Takeshi Fujino \\ Ritsumeikan university \\ Kusatsu, Shiga, Japan \\ fujino@se.ritsumei.ac.jp
}

\begin{abstract}
Deep-learning side-channel attacks (DL-SCAs), applying deep neural networks (DNNs) to SCAs, are known that can easily attack some existing SCA countermeasures such as masking and random jitter. While there have been many studies on profiled DL-SCAs, a new approach that involves applying deep learning to nonprofiled attacks was proposed in 2018. In our study, we investigate the structure of DNN models and attack points (PoI: Points of Interests) for non-profiled DL-SCAs using the ANSSI SCA database with a masking countermeasure. The results of investigations indicate that it is better to use a simple network model, apply regularization to prevent over-fitting, and select a wide range of power traces that contain side-channel information as the PoI. We also implemented AES-128 software implementation protected with the RSM (Rotating Sboxes Masking) countermeasure, which has never been attacked by nonprofiled DL-SCAs, on the Xmega128 microcontroller and carried out non-profiled DL-SCAs against it. Non-profiled DL-SCAs successfully recovered all partial keys while the conventional power analysis could not. We conducted two types of experimental analyses to clarify that DL-SCAs learn mask-values used in the masking countermeasure. One is the gradient visualization used in previous studies, and the other is a new analysis method using partial removal of power traces.
\end{abstract}

\section{CCS CONCEPTS}

- Security and privacy $\rightarrow$ Side-channel analysis and countermeasures.

\section{KEYWORDS}

Side-channel attack (SCA); Deep learning; Non-profiled attacks; AES; RSM Countermeasure

\footnotetext{
Permission to make digital or hard copies of all or part of this work for personal or classroom use is granted without fee provided that copies are not made or distributed for profit or commercial advantage and that copies bear this notice and the full citation on the first page. Copyrights for components of this work owned by others than ACM must be honored. Abstracting with credit is permitted. To copy otherwise, or republish, to post on servers or to redistribute to lists, requires prior specific permission and/or a fee. Request permissions from Permissions@acm.org. ASHES '21, November 19, 2021, Virtual Event, Republic of Korea.

(C) 2021 Association of Computing Machinery.

ACM ISBN 978-1-4503-8662-3/21/11...\$15.00.

https://doi.org/10.1145/3474376.3487285
}

\section{ACM Reference format:}

Kunihiro Kuroda, Yuta Fukuda, Kota Yoshida and Takeshi Fujino. 2021. Practical Aspects on Non-profiled Deep-learning Side-channel Attacks against AES Software Implementation with Two Types of Masking Countermeasure including RSM. In 5th Attacks and Solutions in Hardware Security Workshop (ASHES '21) November 19, 2021, Virtual Event, Republic of Korea. ACM, New York, NY, USA, 12 pages. https://doi.org/10.1145/3474376.3487285

\section{Introduction}

Deep learning using a deep neural network (DNN), a machinelearning technique, has seen the rapid development and been applied to image-recognition systems for surveillance cameras or autonomous vehicles. It has also been put to practical use in security-related applications such as the detection of malicious emails.

Conventional side-channel attacks (SCAs), such as differential power analysis (DPA) [1], correlation power analysis (CPA) [2], and template attack [3], require signal analysis with statistical processing of the power waveform on the basis of the implementation knowledge of the target cryptographic algorithm. Furthermore, high skills are required to analyze cryptographic circuits with SCA countermeasures on which random number masking and/or clock jitter are introduced.

Deep-learning SCAs (DL-SCAs), applying deep learning to SCAs, are proposed [4]. It makes it possible to attack cryptographic circuits protected with conventional SCA countermeasures without knowledge of the countermeasures.

SCAs are classified as profiled or non-profiled. In profiled attacks, a leakage model is constructed using side-channel information from a freely controllable device identical to the target device (profiling device). The attack is then carried out using the constructed leakage model and side-channel information obtained from the target device. One example of profiled attacks is a template attack. Non-profiled attacks use only the side-channel information obtained from the target device. One example of nonprofiled attacks is a CPA. Profiled attacks have been commonly used for DL-SCAs, but non-profiled DL-SCAs were introduced in 2018[5].

\subsection{Related Works}

In 2016, Maghrebi carried out DL-SCAs using convolutional layers for the first time against AES software implementation protected 
with masking countermeasures and showed that the convolution layer is effective against SCAs [4]. Cagli et al. carried out DL-SCAs on AES software and hardware implementation protected with random jitter countermeasure using data augmentation and showed that the attack is possible without the alignment process [6].

Hou carried out a CPA on DPA-ContestV4, which is a dataset of AES software implementation protected with Rotating Sboxes Masking (RSM) countermeasure and successfully attacked it after preprocessing to determine the offset and mask values [7]. Gilmore also successfully attacked DPA-ContestV4 by using a multi-layer perceptron (MLP) [8].

In 2018, Prouff released the ANSSI SCA Database (ASCAD), which is a public dataset of AES software implementation protected with the table re-computation masking countermeasure and carried out profiled DL-SCAs to examine which DNN parameters, such as the number of convolutional layers and filters, are suitable for DL-SCA [9].

In 2018, Timon proposed an attack that applies deep learning to non-profiled attacks (non-profiled DL-SCAs) and showed the effectiveness of the attack against AES software implementation protected with random jitter and masking countermeasure used on ASCAD database. The successful attack was demonstrated on only one partial key against the masking countermeasure and on the limited Points of interest (PoI) after analyzing the leakage point of the mask and the masked intermediate values [5][10]. Other related studies on non-profiled DL-SCAs involved a method using side-channel information from $1 \mathrm{D}$ trace transformed into $2 \mathrm{D}$ image format [11] and an attack against hiding countermeasure based on correlated noise generation [12].

\subsection{Contents of this paper}

In this study, we attacked ASCAD database and our experimental AES-128 software implemented on an Atmel Xmega128 connected to a power-measurement field-programmable gate array using the New AE's ChipWhisperer environment [13]. We carried out nonprofiled DL-SCAs against two types of masking countermeasures; table re-computation masking (used in ASCAD) and RSM, which has not been successfully attacked by non-profiled DL-SCAs so far.

The contributions of this paper are as follows:

1. We provide guidelines on how to select the DL models and attack points on the waveforms (i.e., PoI) to be used in nonprofiled DL-SCAs. We examined several types of structures on MLP and select which is appropriate for non-profiled DLSCAs. Although in the previous study [10], such an attack was carried out on the limited PoI after analyzing the sidechannel leaks, we also show that DL-SCAs are successful on a wide range of sampling points without identifying the limited PoI selected from the prior analysis.

2. The Over-fitting problem has to be avoided for successful DLSCAs. We introduce regularization as a countermeasure against over-fitting and successfully revealed all partial keys against the two masking countermeasures i.e., ASCAD and RSM.
3. We carry out successful non-profiled DL-SCAs against software implemented AES protected with the RSM countermeasure. It should be noted that mask values used in RSM are not used in DL-SCAs. While all 16 partial keys were revealed under 50,000 traces by the DL-SCAs, no partial keys were revealed by the conventional 1st- order CPA.

4. In DL-SCAs, gradient visualization has been used for analyzing the SCA leakage point learned by a model [14]. SHAP, an explainable artificial intelligence, was proposed to explain the reason of output from machine learning [15]. We apply this idea to identify the leak point of mask values by observing the change in attack performance when a part of the input waveform is removed from the selected PoI.

The rest of the paper is organized as follows. We introduce deep learning in Section 2 and introduce the experimental environment and non-profiled DL-SCAs in Section 3. In Section 4, we present the guidelines for non-profiled DL-SCAs and present the results from carrying out non-profiled DL-SCAs on RSM countermeasure AES in Section 5. In Section 6, we discuss the side-channel leakages learned by the network model used in non-profiled DLSCAs.

\section{Deep Learning Techniques}

\subsection{Structure of neural network}

A neural network consists of several layers of neurons. A neuron is the mathematical model of a neuron in the human brain. A neural network consists of a pair of input and output layer, and several intermediate layers placed between those layers. This structure is called an MLP. Neural networks for classification tasks are trained by a training dataset $\mathrm{D}=\left\{\left(x_{i}, y_{i}\right)\right\}_{i=1}^{N}$ which is a set of $\mathrm{N}$ pairs of a finite number of inputs $\mathrm{x}$ and correct labels $\mathrm{y}$. The network learns to minimize the error between the correct label y and the predicted value $\hat{y}$ and obtains the ability to discriminate.

\subsection{Over-fitting and validation accuracy}

Over-fitting refers to a situation in which the network adapts too much to the training dataset and fails to classify an unknown data that is different from the training data. During training, we observe the classification accuracy and loss for the validation dataset (unknown dataset for the network) to monitor over-fitting. Regarding over-fitting, classification accuracy for the validation dataset (hereafter, validation accuracy) becomes lower than that for the training dataset (hereafter, training accuracy). Similarly, when over-fitting is in progress, the classification loss for the training dataset decreases but the loss for the validation dataset increases. In the following experiments, we used validation accuracy to evaluate the trained model.

\subsection{Regularization of weights in DNNs models}

Regularization is a method for preventing over-fitting. It should improve the generalization performance of network models. From the experiments introduced in Sections 4 and 5, we found that regularization has a significant advantage on the performance of non-profiled DL-SCAs compared to results without it. 
L1 regularization, L2 regularization, and L1/L2 regularization, which applies both L1 and L2 regularization, are mainly used in deep learning. When regularization is not used, the network learns to minimize the loss function $\mathrm{L}(\mathrm{x})$. If $\mathrm{L} 1$ regularization is used, weight parameters $w_{i}$ used on DNNs are determined to minimize the sum of the loss function $L^{\prime}(x)$, which includes the L1 regularization term as a penalty in addition to loss function $L(x)$, as shown in Equation (1).

$$
L^{\prime}(x)=L(x)+\lambda \sum_{i=0}^{n}\left|w_{i}\right|
$$

$\lambda$ in Equation (1) is the L1 regularization parameter that adjusts the strength of the regularization.

Similarly, if L2 regularization is used, $w_{i}$ is determined to minimize $L^{\prime}(x)$, which includes the $\mathrm{L} 2$ regularization term as a penalty in addition to loss function $L(x)$, as shown in Equation (2).

$$
L^{\prime}(x)=L(x)+\lambda \sum_{i=0}^{n}\left|w_{i}\right|^{2}
$$

$\lambda$ in Equation (2) is the L2 regularization parameter that adjusts the strength of the regularization.

If $\mathrm{L} 1 / \mathrm{L} 2$ regularization is used, $w_{i}$ is determined to minimize the $L^{\prime}(x)$, which includes the L1/L2 regularization term as a penalty in addition to loss function $L(x)$, as shown in Equation (3).

$$
L^{\prime}(x)=L(x)+\lambda_{1} \sum_{i=0}^{n}\left|w_{i}\right|+\lambda_{2} \sum_{i=0}^{n}\left|w_{i}\right|^{2}
$$

$\lambda_{1}$ and $\lambda_{2}$ in Equation (3) are the L1 regularization parameter and L2 regularization parameter respectively.

Therefore, the sum of $w_{i}$ is evaluated to prevent the weights from becoming too large and avoid over-fitting

\section{Experiments on non-profiled DL-SCAs}

\subsection{Non-profiled DL-SCA procedure}

The non-profiled DL-SCA is an attack that applies deep learning to non-profiled attacks that use side-channel information obtained only from the target device [10].

First, the attacker obtains $\mathrm{N}$ power-consumption waveforms $T_{i}(1 \leq \mathrm{i} \leq \mathrm{N})$ corresponding to $\mathrm{N}$ plaintexts $p_{i}(1 \leq \mathrm{i} \leq \mathrm{N})$ in cryptographic operations with a fixed key $k_{\text {secret }}$. In the case of byte-by-byte attacks against AES, the attacker also prepares network models $M_{k}\left(0 \leq \mathrm{k} \leq 2^{8}-1\right)$ for each key candidate $\mathrm{k}$. Each model is trained to output the specific intermediate value (label) $h_{k, i}$ computed from the key candidate $\mathrm{k}$ and the plaintext $p_{i}$ $(1 \leq \mathrm{i} \leq \mathrm{N})$. In the experiments, the least significant bit (LSB) of the $\mathrm{S}$ Box output value in the first round of AES-128 is used as the output label. Other labelling methods (such as MSB, HW labeling) are available, but we have not used them in the later experiments. The key $k^{*}$ corresponding to the model $M_{k^{*}}$ with the highest accuracy after the training is specified as the correct key. The procedure of the non-profiled DL-SCA is shown in Algorithm1. In all experiments, the optimizer was ADAM, the learning rate was 0.001 , batch size was 1000 , the number of epochs was 100 , and the loss function was the mean square error. These hyperparameters are same ones which used in previous study [10].

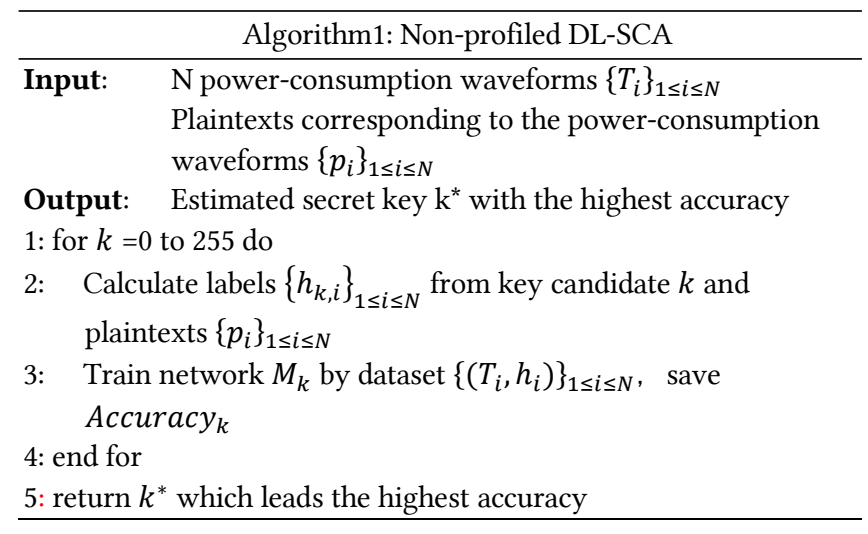

\subsection{Waveform datasets used in the experiments}

We carried out non-profiled DL-SCAs against AES-128 software implementation protected with the table re-computation masking and RSM countermeasures. The two datasets used in the experiments are as follows.

(1) ASCAD (countermeasure: table re-computation masking) [8]

ASCAD is a dataset that provides electromagnetic (EM) emission waveforms during operation of AES software implementation protected with the table re-computation masking countermeasure on the 8-bit AVR ATMega8515. The mask values for 0th and 1st bytes are set to 0 , so 0 th and 1 st bytes are regarded as unprotected. The EM waveforms are measured using a digital oscilloscope with a sampling rate of $2 \mathrm{GS} / \mathrm{s}$. ASCAD discloses waveforms of 2 nd byte SubBytes processing in 1st round as the PoI for the 2nd byte attack.

(2) In-house Dataset measured by ChipWhisperer

(countermeasure: RSM)

The DPAContestV4.2 dataset is available as a public dataset for AES software implementation protected with the RSM countermeasure [16]. However, this dataset is not suitable for nonprofiled DL-SCAs evaluation because the key is changed every 5000 waveforms. Therefore, we prepared an in-house dataset by using Atmel 8-bit microcontroller Xmega128. The source code is modified from that available on the DPAContest website. The power consumption during the AES processing was measured using an A/D converter (sampling rate: $29 \mathrm{MS} / \mathrm{s}$ ) implemented in ChipWhisperer-Lite [13]. The experimental condition for waveform acquisition is shown in Fig. 1.

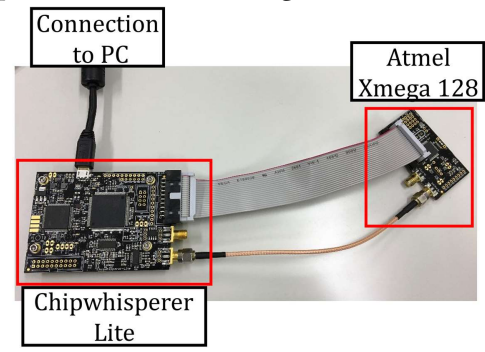

Figure 1: Experimental condition for acquiring power consumption waveform on AES processing 


\section{Guidelines for non-profiled DL-SCAs}

In this section, we present the results of attacks on the 2nd byte of ASCAD to provide some guidelines on how to select DNN models and attack points on waveforms for non-profiled DL-SCAs. We discuss the performance of the attack on various numbers of layers and nodes on a network model (MLP) and whether it is necessary to select a small PoI after analyzing the leakage points of intermediate values. We also discuss how to improve the attack by regularization, which is a countermeasure against over-fitting problem on DNNs.

\subsection{Table re-computation masking countermeasure}

The table re-computation masking countermeasure, applied in ASCAD, prevents side-channel leakage by using an S-Box table computed with two random numbers. This masked S-Box is used in the SubBytes layer because simple additive masking cannot be applied on a nonlinear transformation [17][18]. Figure 18 in Appendix shows an overview of the first round of AES protected with the table re-computation masking countermeasure. The masked S-Box is pre-computed using 1-byte random mask values $m_{\text {in }}$ and $m_{\text {out }}$, as shown in the figure. The input plaintext is also masked by a 16-byte random value $m_{1}$. Since the values of all nodes are masked by random masks which unknown to the attacker, leakages about $p \oplus k_{1}$ and S-Box $\left(p \oplus k_{1}\right)$ do not appear in the side-channel information and cannot be attacked by a conventional 1st-order CPA. The $m_{1}, m_{\text {in }}$, and $m_{\text {out }}$ will be changed when the next plaintext is encrypted. Non-profiled DLSCAs are performed using the method shown in Subsection 3.1, and the assumption is that the attacker does not know that a masking countermeasure is implemented at the target. Therefore, we train DNNs $M_{k}(0 \leq \mathrm{k} \leq 255)$ by the LSB on the output of S$\operatorname{Box}\left(p \oplus k_{1}\right)$ in the first round, which is computed only from the key candidate $k$ and the plaintext $p_{i}(1 \leq \mathrm{i} \leq \mathrm{N})$. By monitoring validation accuracy on the training, we estimate the key candidate $k^{*}$ corresponding to $M_{k^{*}}$ with the highest accuracy as the correct key.

\subsection{Layers and nodes in network model}

We used the MLP with two intermediate layers (20 nodes in the first layer and 10 nodes in the second layer) used in the previous work [10] as a reference. We also observed the change in attack performance when the number of intermediate layers and nodes are varied to identify the appropriate network in our study. The convolutional layer is effective, mainly against random jitter countermeasure [6], so we did not use it. However, since it is also effective to some extent for masking countermeasures [4], researches using convolutional layers will be future works.

We first verified the change of attack performance depending on the number of intermediate layers of the MLP. The number of intermediate layers with 20 nodes was changed from 2 to 8 , and attacks were carried out against the 2nd byte of ASCAD. We used
30,000 waveforms (training waveforms: 24,000, validation waveforms: 6,000). We observed the difference in validation accuracy between the correct key and the incorrect key with the highest accuracy as an evaluation metric. Figure 2 (a) shows the difference in this accuracy when the number of intermediate layers was changed. Although the number of layers increased, the accuracy of the correct label did not improve compared with the result with two layers.

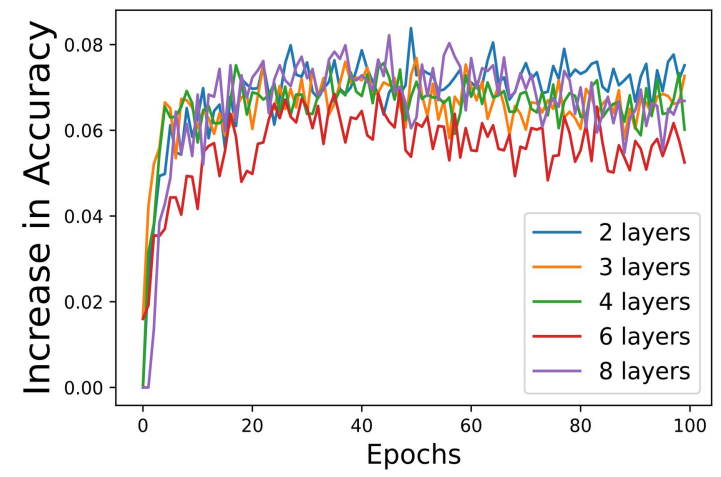

(a) Dependence on the number of intermediate layers (The number of nodes in each layer is fixed to 20)

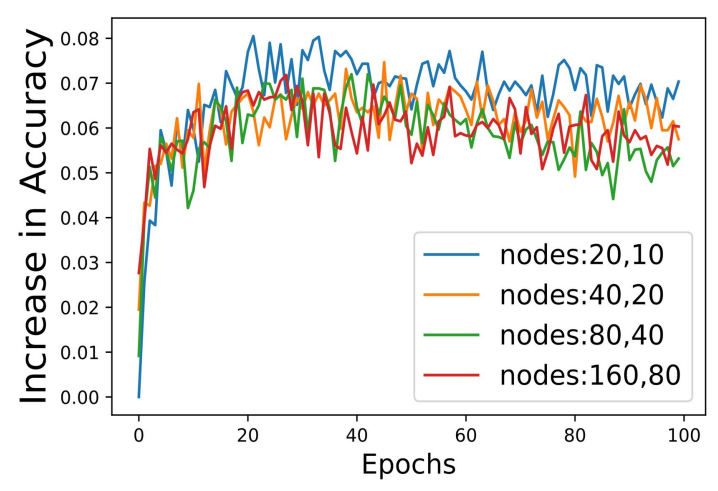

(b) Dependence on the number of nodes (The number of intermediate layers is fixed to 2)

Figure 2: Dependence on the number of nodes and intermediate layers with the validation accuracy in attack against ASCAD 2nd Byte

We then verified the change in attack performance with the number of nodes in the intermediate layer of the MLP. The number of layers was fixed to 2. Using the same evaluation method as in Fig. 2 (a), Fig. 2 (b) shows the difference in accuracy of the correct label when the number of nodes varied. As in the case of changing the number of layers, no significant difference was observed when the number of nodes increased.

In a previous study [10], it was stated that the use of complex networks may improve the performance of non-profiled DL-SCAs, but the complexity of the network did not make a significant difference according to our experimental results. Therefore, considering additionally that the more complex the network is, the 
longer it takes to learn, we also used the same network with two intermediate layers (number of nodes: 20, 10) as previous study [10] in the subsequent experiments.

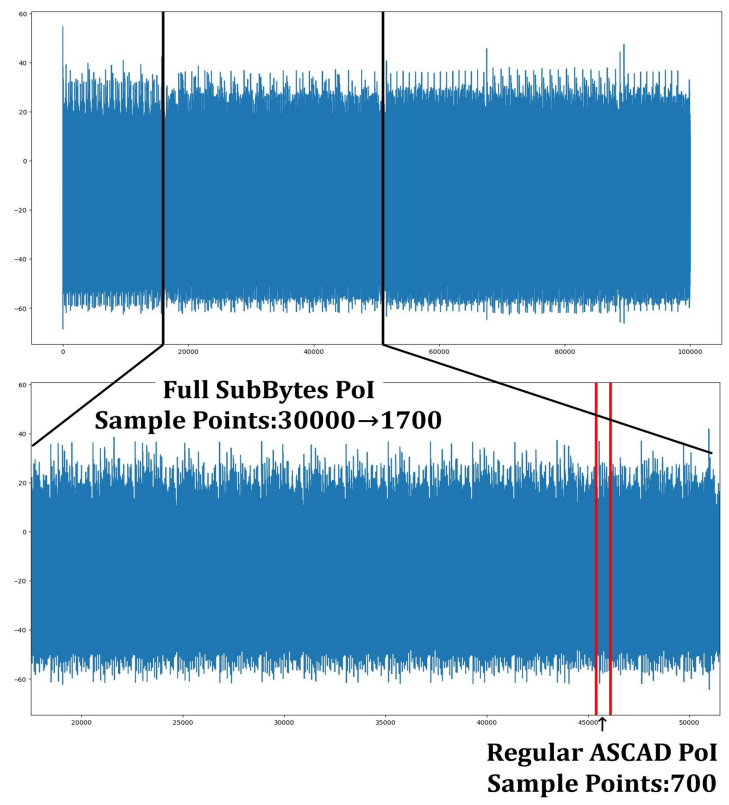

Figure 3: The raw waveform of ASCAD and the attack points used in the attack (Regular ASCAD and Full SubBytes in the first Round)

\subsection{How to choose Attack Point (PoI)}

In this section, we discuss how the attack performance changes depending on the choice of PoI. In ASCAD, the mask values $m_{1}$, $m_{\text {in }}$, and $m_{\text {out }}$ are used to identify the 2nd-byte SubBytes processing point in the first round, which is extracted from the side-channel information. Assuming a realistic attack scenario where the attacker does not have any prerequisite knowledge of the target, it is reasonable to select the point where all SubBytes on the first round of AES are processed from the raw waveform of ASCAD as shown in Fig. 3. We examined whether the attack performance changes when the attack point is the 2nd byte processing point specified in ASCAD (Regular ASCAD PoI in Fig. 3, sample points: 700) or when the attack point is the entire SubBytes processing point (Full SubBytes PoI in Fig. 3, sample points: 34000). When full SubBytes processing area is selected as an attack point, the number of input samples is reduced to 1700 by averaging every 20 points in order to reduce the number of input samples. We used 30,000 waveforms (training waveforms: 24,000, validation waveforms: 6,000) to carry out the attack. Figure 4 (a) shows the validation accuracy of each estimated key when the attack point was the regular ASCAD PoI. Figure 4 (b) shows the validation accuracy when the attack point was the full SubBytes PoI. The attack succeeded even when the attack point was wider. The larger number of the attack points, the larger the number of epochs required for estimating the correct key, but the final accuracy is slightly higher with a larger number of attack points. In the attack on ASCAD, described in detail in Section 6, the network learns the leakage of $m_{\text {out }}$. This mask value was used in the SubBytes process of all bytes, so the Full SubBytes PoI provides more hints (leaks of $m_{\text {out }}$ ) to the model. Therefore, the final accuracy is likely to be higher if the Full SubBytes PoI is used as the attack point.

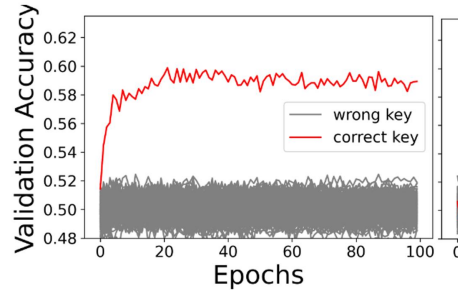

(a) Regular ASCAD PoI

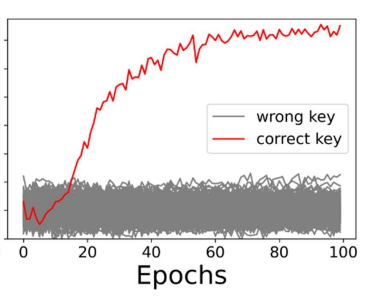

(b) Full SubBytes PoI
Figure 4: Results of validation accuracy attack on 2nd byte when using Regular ASCAD PoI and Full SubBytes PoI (validation accuracy per estimated key)

\subsection{Effect of regularization}

In Subsection 4.3, we showed that an attack against the 2nd byte of ASCAD is possible even if the attack point is widely selected by including SubByte processing of all bytes (Full SubByte PoI) in the first round. Since this PoI involves SubBytes processing of all bytes, we used the same 30,000 waveforms to attack all bytes. We observed the validation accuracy and found that we could recover 15 partial keys, but we could not successfully obtain the 3rd partial key.

The fact that the validation accuracy of model trained by dataset of the correct key did not increase suggests that the trained network model does not have generalization performance. To increase validation accuracy (i.e., to improve generalization performance), we applied the regularization of weight parameters on DNNs, as described in Subsection 2.2. We tried several regularization parameters and adopted the L1/L2 regularization shown in Equation (3), which achieves the best result. Regularization parameters $\left(\lambda_{1}: 0.00001, \lambda_{2}: 0.00001\right)$ were set for 1 st dense layer and $\left(\lambda_{1}: 0.0008, \lambda_{2}: 0.0008\right)$ for 2 nd dense layer, and the attack was conducted again.

Figure 5 shows the validation accuracy for the 3rd byte attack without (a) and with (b) regularization. Compared (a) with (b), the accuracy of the model trained with regularization was clearly higher, indicating the effectiveness of regularization.

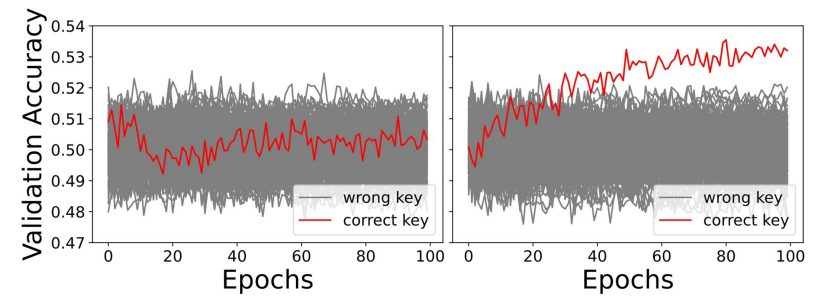

(a) without regularization

(b) with regularization

Figure 5: Validation accuracy of each estimated key for attack on 3rd byte of ASCAD 


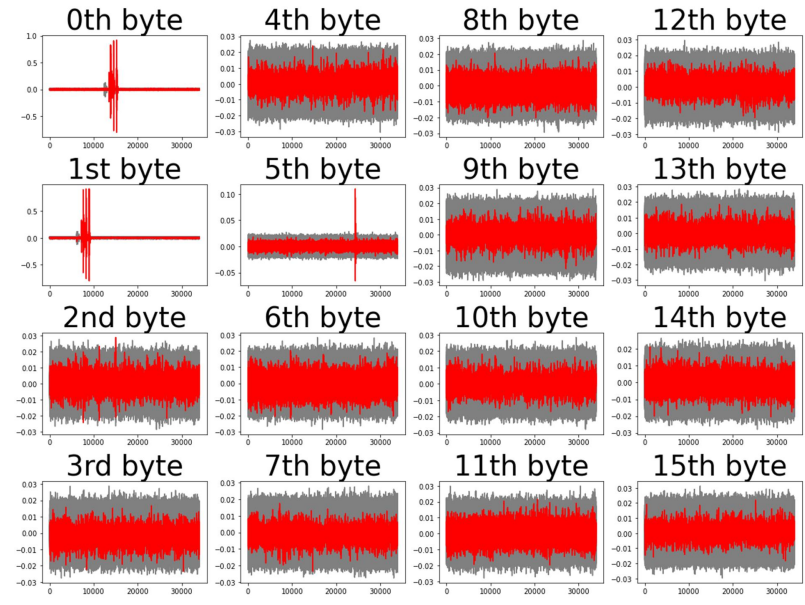

Figure 6: The result of CPA against all bytes of ASCAD. Vertical axis means the correlation, and horizontal axis means the sampling point.

\subsection{Comparison with 1st-order CPA}

A CPA is a typical and powerful attack in non-profiled attacks. The results of the 1st-order CPA with 30,000 waveforms, which is the same number of waveforms as DL-SCAs we carried out, are shown in Fig. 6. The CPA was carried out by applying Hamming Weight $(\mathrm{HW})$ model on the output value S-Box $\left(p \oplus k_{1}\right)$ in the first round of AES. Figure 7 also shows the results of non-profiled DL-SCAs with regularization we carried out against all ASCAD bytes.

Both CPA and DL-SCAs succeeded on the 0th and 1st bytes on which the masking countermeasure was disabled. For 5th byte, the correlation value of the correct key was the highest, and all other bytes had no correlation peak. Therefore, the CPA against the masking countermeasure almost failed. Non-profiled DL-SCAs with regularization succeeded in the 2nd and subsequent bytes, where the masking countermeasure was effective even without the knowledge that the masking countermeasure was applied.

Figure 8 shows the number of partial keys successfully recovered when the number of waveforms increased to 30,000 . The CPA was not successful, except for the 3 bytes, which was not masked, and 5th byte. On the other hand, non-profiled DL-SCAs without regularization successfully attacked 15 bytes in 30,000 waveforms, and all 16 bytes in 25,000 waveforms when regularization was applied. These results mean that non-profiled DL-SCAs had higher attack performance than the typical (1st order) CPA. Against the masking SCA countermeasure described in this section, the attack is still possible if the internal mask value can be estimated using a high-order CPA by the conventional method. The fact that an attacker who does not know the internal processing of the countermeasure can still obtain all partial keys using non-profiled DL-SCAs suggests that such an attack increases the threat of side-channel attacks.

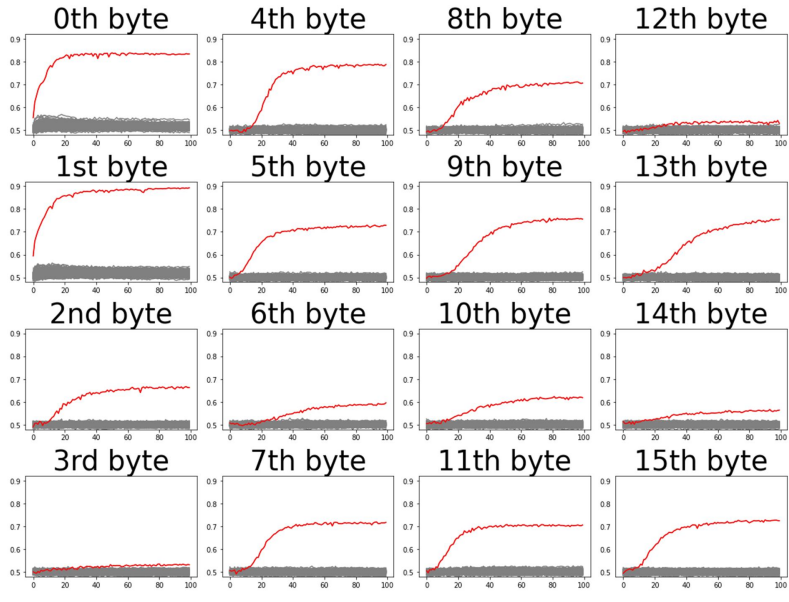

Figure 7: The result of non-profiled DL-SCAs against all bytes of ASCAD. Vertical axis means the validation accuracy, and horizontal axis means the number of epochs.

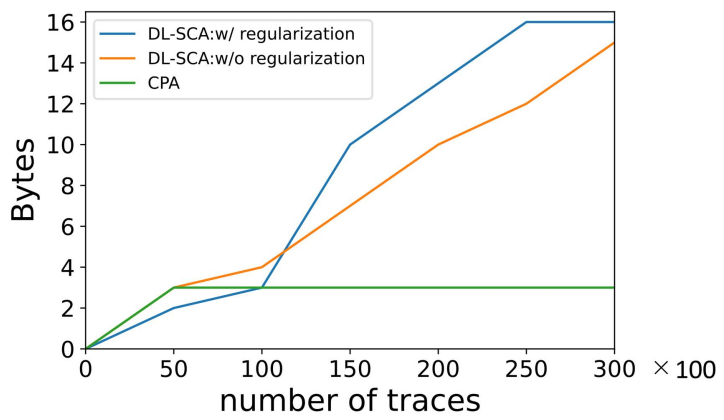

Figure 8: Comparison of attack results between nonprofiled DL-SCAs with and without regularization and CPA (successfully recovered partial keys per number of waveforms)

\section{Non-profiled DL-SCAs against AES-128 RSM}

\subsection{Implementation of RSM countermeasure}

The RSM (Rotating Sboxes Masking) countermeasure uses 16 types of $\left\{S-b o x_{i}^{\prime}\right\}_{0 \leq i \leq 15}$ pre-computed with 16-byte fixed mask values $\mathrm{m}[\mathrm{i}](0 \leq \mathrm{i} \leq 15)$ as the $\mathrm{S}$-Box used in SubBytes processing. Before and after the S-Box transformation, masking is executed on the intermediate values with $\mathrm{m}[\mathrm{i}]$ to prevent side-channel leakage [19]. The 16 types of S-Boxes used in encryption are calculated using equation (4).

$$
S-\operatorname{Box}_{i}^{\prime}(x)=S-B o x(x \oplus m[i]) \oplus m[(i+1) \bmod 16]
$$




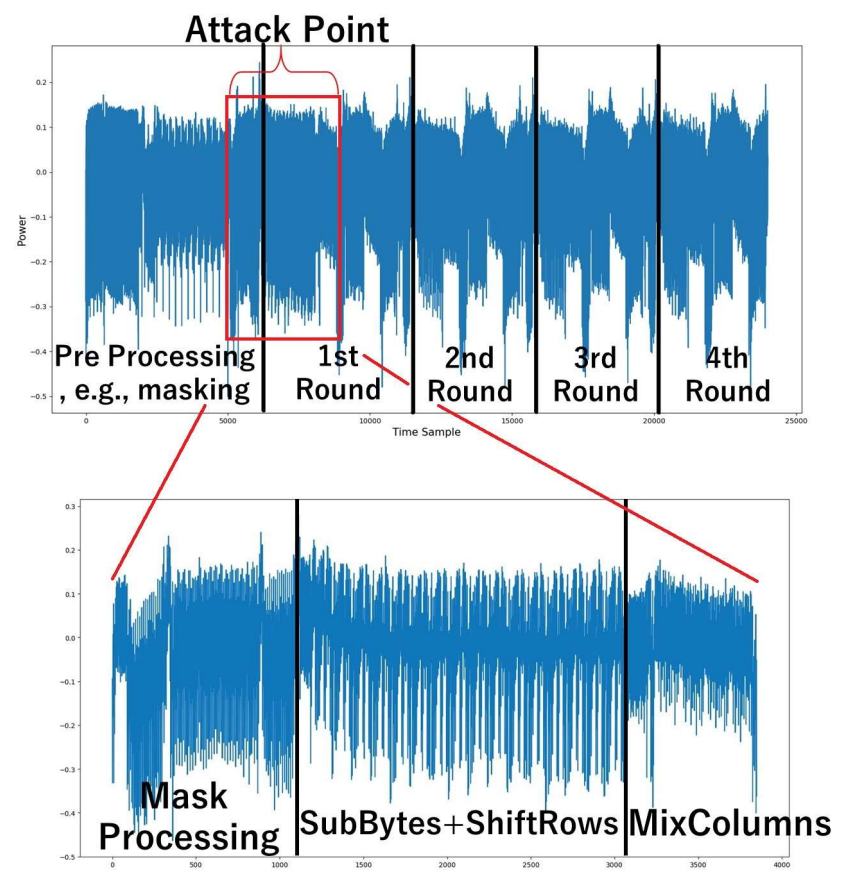

Figure 9: Power-consumption waveforms we collected and PoI

Figure 19 in Appendix shows an overview of the first round of AES protected with the RSM countermeasure. The offset that appears in the figure is a parameter that specifies which of the 16 $\mathrm{S}$-Boxes and mask values should be used in the first round of processing for each byte. Thus, the possible values of offset are from 0 to 15. The S-Box used in the SubBytes layer is rotated in each round. The fixed mask value $\mathrm{m}[0: 15]=[0 \mathrm{x} 03,0 \mathrm{x} 0 \mathrm{c}, 0 \mathrm{x} 35$, 0x3a, 0x50, 0x5f, 0x66, 0x69, 0x96, 0x99, 0xa0, 0xaf, 0xc5, 0xca, 0xf3, 0xfc] used in our experiment is the same as that used in DPAContestV4.2[16].

\subsection{PoI determination on power traces}

We experimented with the program of AES software implementation protected with the RSM countermeasure published by DPA ContestV4.2, excluding the shuffling countermeasure of the byte processing.

We collected 50,000 (training waveforms: 40,000, validation waveforms: 10,000) power-consumption waveforms by using the experimental environment described in Subsection 3.2. The power consumption waveform is shown in Fig. 9.

When carrying out non-profiled DL-SCAs, we assumed that the attacker does not have knowledge of the AES implementation techniques in the target device. Therefore, the fixed 16-byte mask value on RSM is not used in the attack because the attacker does not know that the RSM countermeasure is in place. We also assumed that the attacker can roughly select the powerconsumption waveform, so we used all regions including the preprocessing (mask processing) and SubBytes, ShiftRows, and
MixColumns processing in the first Round as the PoI (sample points of clipped waveform:3850). It is possible that the attacker does not include the mask processing part in the PoI, but we will discuss such a case in Subsection 6.2.

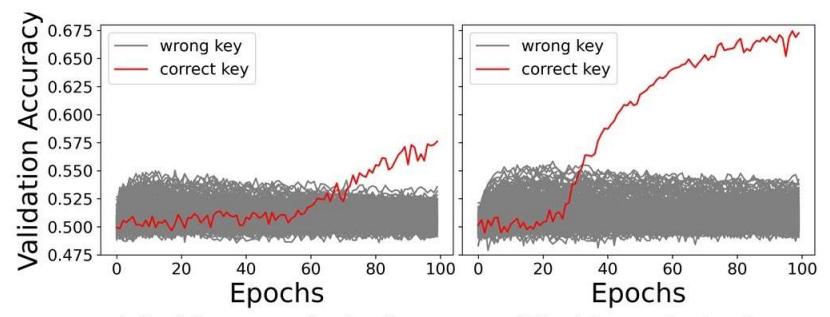

(a) without regularization

(b) with regularization

Figure 10: Validation accuracy of each estimated key for RSM 1st byte attack

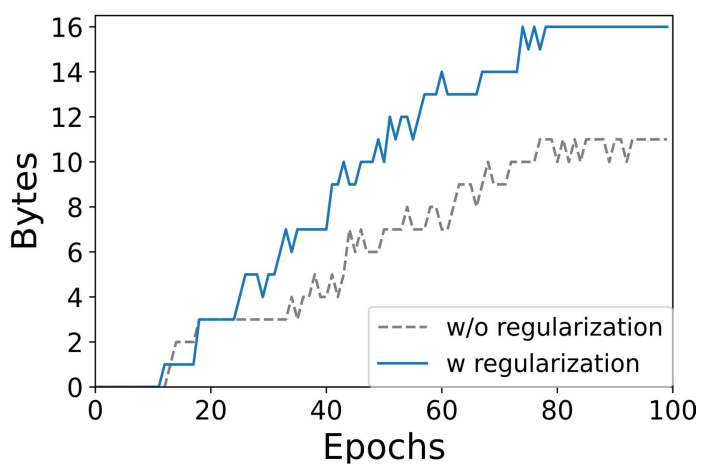

Figure 11: Comparison of attack results between nonprofiled DL-SCAs with and without regularization (successfully recovered bytes per epoch)

\subsection{Results of non-profiled DL-SCAs against AES with RSM countermeasure}

As with the experiments discussed in Section 4, we carried out non-profiled DL-SCAs using the method discussed in Subsection 3.1. We learned the MLPs using the LSB on the S-Box $\left(p \oplus k_{1}\right)$ in the first round, which is computed from only key candidate $\mathrm{k}$ and plaintext $p_{i}(1 \leq \mathrm{i} \leq \mathrm{N})$. The key candidate with the highest accuracy was estimated as the correct key. To show the effect of regularization on the RSM countermeasure, we conducted attacks both with and without regularization (regularization parameters were the same as those discussed in Subsection 4.4). Figure 10 shows the validation accuracy for each estimated key with and without regularization when we attack the 1 st byte. The accuracy of the model which learned the correct key dataset with regularization was much higher than that without regularization. Figure 11 shows the relationship between the number of revealed partial keys and that of epochs on learning. When regularization was not applied, only 10 out of 16 bytes were successfully attacked. By applying regularization and observing validation accuracy, we found that all the partial keys were successfully revealed, which indicates the effectiveness of non-profiled DLSCAs against the RSM countermeasure. 


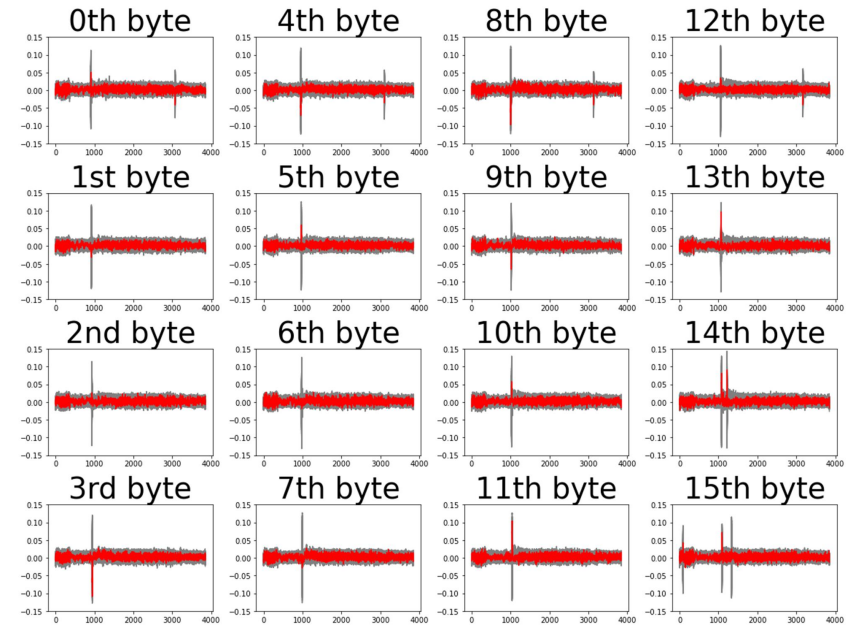

Figure 12: Results of CPA against RSM for all bytes. Vertical axis means the correlation, and horizontal axis means the sampling point.

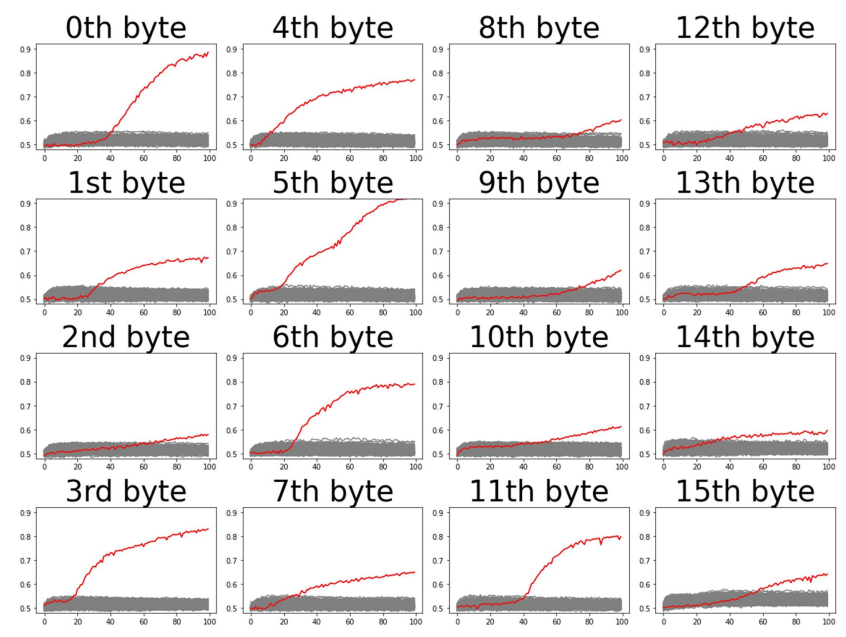

Figure 13: Result of non-profiled DL-SCAs against RSM for all bytes. Vertical axis means the validation accuracy, and horizontal axis means the number of epochs.

\subsection{Comparison with 1st-order CPA}

Using the same HW model as in Subsection 4.5, the results of the CPA with 50,000 waveforms, the same number of waveforms as with non-profiled DL-SCAs we carried out, are shown in Fig. 12. The results of non-profiled DL-SCAs with the regularization applied against the RSM countermeasure performed in Subsection 5.3 are also shown in Fig. 13.

The CPA results contain a peak, but the correlation with the incorrect key has the highest value, and the attack failed for all bytes. The results for non-profiled DL-SCAs are shown in Fig.13. The attacks against all partial keys were successful. It suggests that an attack against the RSM countermeasure (as well as ASCAD) is possible even if the attacker does not know that the countermeasure was implemented in the target and the internal processing of mask values, etc.

\section{Discussion of the model's learning points}

\subsection{Evaluation with the gradient visualization}

To determine whether the network learns the leaks, we introduce Equation (5). In the literature [14], it is described as gradient visualization.

$$
\nabla T_{i}=\frac{\partial L_{T}}{\partial x_{i}}
$$

In Equation (5), $L_{T}$ denotes the loss when waveform $\mathrm{T}$ is input to the model and $x_{i}$ denotes the $\mathrm{i}$-th sample variable of waveform $\mathrm{T}$.

We apply Equation (5) to the model trained by the attack against ASCAD using regularization mentioned in Subsection 4.4. To analyze what the network has learned on using gradient visualization, it is necessary to understand what operations are being executed in which parts of the waveform. For this reason, the correlation between the mask value and power-consumption waveform, and the correlation between the masked intermediate value and the power-consumption waveform were obtained. The upper waveform of Fig. 14 shows the correlations between the power-consumption and LSB of the following values:

Blue) 2nd-byte Mask value: $m_{1}$

Orange) 2nd-byte Masked S-box output masked by $m_{1}$ : $\mathrm{S}-\operatorname{Box}\left(p \oplus k_{1}\right) \oplus m_{1}$

Green) Mask value: $m_{\text {out }}$

Black) 2nd-byte Masked S-box output masked by $m_{\text {out }}$ : S-Box $\left(p \oplus k_{1}\right) \oplus m_{\text {out }}$

It can be concluded that the operations using these values are carried out where the correlation peaks appear. The lower waveform of Fig. 14 shows the gradient visualization $\nabla T_{i}$ at each sample point when the validation data waveform is input to the network trained with the training dataset of the correct key for the 2nd-byte attack. Since the peak locations of $\nabla T_{i}$ overlap the peak locations of the four correlations of $m_{1}, m_{\text {out }}$, S-Box $\left(p \oplus k_{1}\right) \oplus$ $m_{1}$, and $\mathrm{S}-\operatorname{Box}\left(p \oplus k_{1}\right) \oplus m_{\text {out }}$, it seems that the network learns the leakage of the two sets of mask and masked S-Box output values. Figure 20 in Appendix shows an overview of the processing of the attack points during an attack on the table recomputation masking countermeasure in Subsection 4.4. From Fig.14 and Fig. 20, we can consider that by XORing $m_{\text {out }}$ with S$\operatorname{Box}\left(p \oplus k_{1}\right) \oplus m_{\text {out }}$, which is the output of the pre-computed SBox', the LSB of the correct label $\operatorname{S}-\operatorname{Box}\left(p \oplus k_{1}\right)$ is derived. Similarly, learning $\mathrm{S}-\operatorname{Box}\left(p \oplus k_{1}\right) \oplus m_{1}$ and $m_{1}$ can also deliver the correct label $\operatorname{LSB}\left(\mathrm{S}-\operatorname{Box}\left(p \oplus k_{1}\right)\right)$.

We conducted the same analysis for the attack against AES protected with the RSM countermeasure. The upper part of Fig. 15 shows the correlations between the power-consumption and LSB of the following values:

Blue) 1st-byte Mask value $m[$ offset $[1]+1]$

Orange) 1st-byte Masked S-box output: $\operatorname{S-Box}\left(p \oplus k_{1}\right) \oplus m[$ offset $[1]+1]$ 


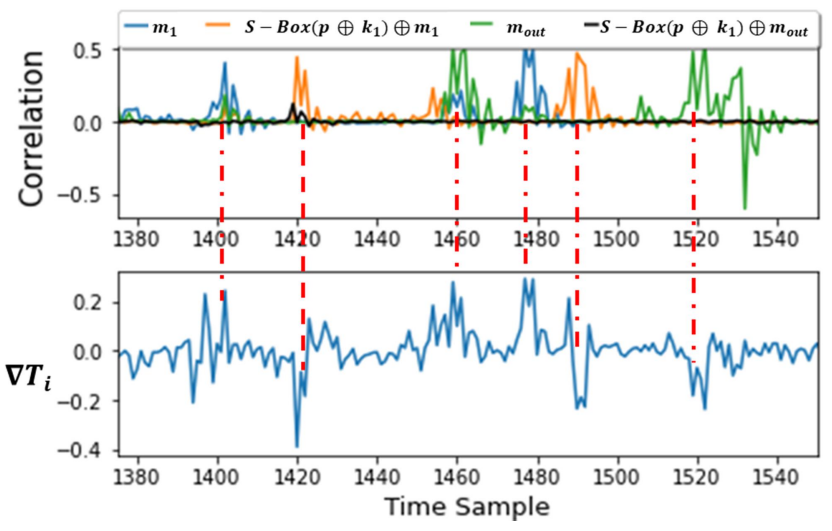

Figure 14: Correlation of mask and masked S-Box output (top) and $\nabla T_{i}$ (bottom) (using 2 nd byte of ASCAD)
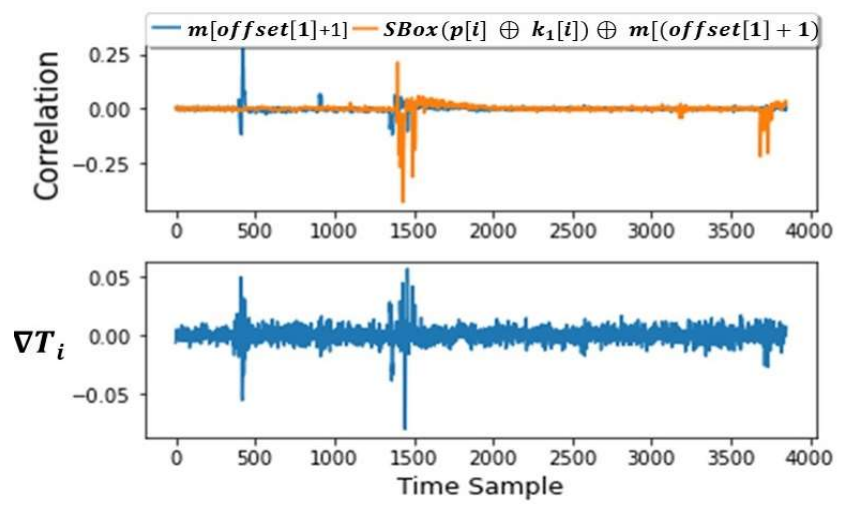

Figure 15: Correlation of masked and masked S-Box output (top) and $\nabla T_{i}$ (bottom) (using 1st byte of RSM countermeasure dataset)

The lower part of Fig. 15 shows $\nabla T_{i}$ when the validation data are input to the network trained with the training data set of the correct key for the 1st-byte attack. From Fig. 15, it appears that models are learning the leakages of $\operatorname{S-Box}\left(p \oplus k_{1}\right) \oplus$ $m[$ offset $[1]+1]$, and $m[$ offset $[1]+1]$. Figure 21 in Appendix shows an overview of the processing of the attack points during an attack on the RSM countermeasure in Subsection 5.3. From Fig. 21 , there is only one masked S-Box output value $\operatorname{S}-\operatorname{Box}\left(p \oplus k_{1}\right) \oplus$ $m[$ offset $[1]+1]$, which appears during the cryptographic process of the first round.

From the above results, in non-profiled DL-SCAs against both masking countermeasures, the network model learns the mask value $m$ and the masked output S-Box value S-Box $\left(p \oplus k_{1}\right) \oplus m$ from the power consumption value. The network model unmasked the learned masked S-Box output value using the mask value and estimated the LSB of unmasked S-Box output value LSB(S$\left.\operatorname{Box}\left(p \oplus k_{1}\right)\right)$, which was set as the label.

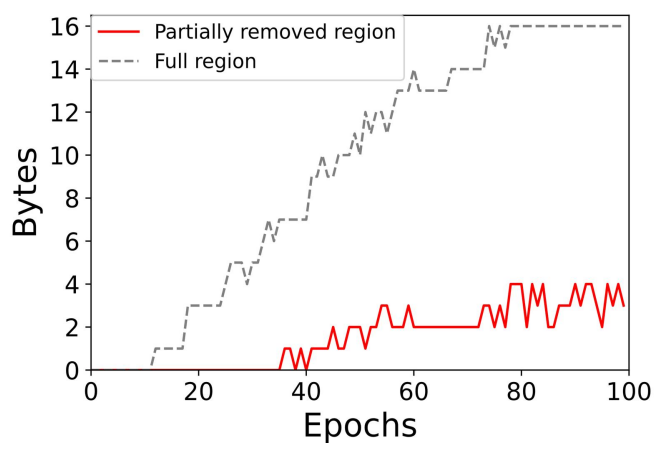

Figure 16: Results of non-profiled DL-SCAs against AES software implementation protected with RSM countermeasure when the waveform on mask processing is partially removed
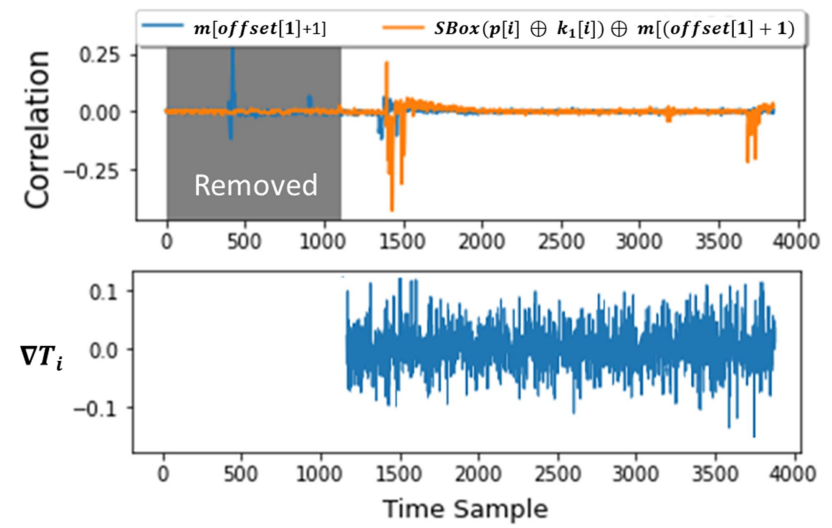

Figure 17: Correlation of masked and masked S-Box output (top) and $\nabla T_{i}$ (bottom) on RSM (0-1100 points are removed)

\subsection{Evaluation with partial removal of power traces}

Research is progressing on approaches, such as SHAP, that explain the output of machine learning [14]. As a simple approach, we observe the change of attack performance depending on whether the mask leak is included or excluded from the PoI. Therefore, we verify whether the model, in fact, learns the leakage points of the mask in non-profiled DL-SCAs. We removed the sample before 1100 points corresponding to the "mask processing" (as shown in Fig. 21 in Appendix) from the attack point discussed in Subsection 5.2. Therefore, the attack points were only SubBytes, ShiftRows, and MixColumns processing points. We applied regularization, as in Subsection 5.3, and conducted the attack. Figure 16 shows the number of successfully recovered partial keys per epoch by observing validation accuracy.

In contrast to the results of the attack including masking processing point mentioned in Subsection 5.3 at epoch 100, only 3 out of 16 partial keys were successfully attacked when validation accuracy was observed. Figure 17 also shows the $\nabla T_{i}$ derived in the same manner as mentioned in Subsection 6.1. In contrast to 
Fig. 15, when the mask processing area was excluded from the PoI, no significant peak appeared in $\nabla T_{i}$ indicating that the model is not able to effectively learn side-channel leakages.

These results confirm that the model learns the leakage of the mask, and the leakage points of the mask need to be included in the PoI in DL-SCAs. The RSM countermeasure does not use mask values in the SubBytes processing. Therefore, we need to choose a wide range of attack points including masking processing since non-profiled DL-SCAs are possible even with a wide range of attack points, as discussed in Subsection 4.3.

\section{Conclusion}

We reported on the results of non-profiled DL-SCAs against AES software implementation protected with two types of masking countermeasures. One is the table re-computation masking countermeasure, and the other is the RSM countermeasure.

From the results of the attacks against the above two types of masking countermeasures, the practical guidelines on non-profiled DL-SCAs, and the analysis of leakage points are clarified as follows.

1. The MLP network model with two intermediate layers with 20 and 10 nodes is sufficient for such attacks. There is no need to increase the number of layers and nodes according to the experimental results on ASCAD.

2. The selected range of waveforms to be learned, i.e., the PoI, can be attacked by specifying a large range that includes the S-Box processing of all bytes and does not need to be divided into byte-by-byte computational regions.

3. Applying L1/L2 regularization to the weight parameters on DNNs during non-profiled DL-SCAs improves the performance of DL-SCAs and allows us to recover all partial keys for both masking countermeasures.

4. By analyzing the information learned by the network model using gradient visualization, we find that the network model learns the mask value $m$ and the masked S-Box output value S- $\operatorname{Box}\left(p \oplus k_{1}\right) \oplus m$ from the side-channel information. DNNs infer the correct label $\operatorname{S-Box}\left(p \oplus k_{1}\right)$ from these values, so the accuracy of the correct key will be increased.

5. As a new method to evaluate what the model learned, the performance of non-profiled DL-SCAs get worsens when the arithmetic part that uses the mask value $m$ in RSM implementation is removed from the PoI. This confirms that the mask value $m$ is being estimated from the waveform.

Non-profiled DL-SCAs against AES software implementation protected with a masking countermeasure does not require any special attacking method (e.g., mask-value estimation before attacking, as used in the conventional 2nd-order CPA) to recover all partial keys. Attackers do not need to refine the PoI of the acquired waveform. Therefore, we can conclude that the threat of a realistic side-channel attack has increased since such an attack was successful without knowledge of the side-channel countermeasure implementation used by the target. In the future, non-profiled DL-SCAs will become more common for the evaluation of implemented side-channel attack countermeasures. It will also be necessary to study implementation schemes that are more resistant to DL-SCAs.

\section{ACKNOWLEDGMENTS}

This work was supported by JST-Mirai Program Grant Number JPMJMI19B6, Japan.

\section{REFERENCES}

[1] Kocher, Paul, Joshua Jaffe, and Benjamin Jun. 1999. Differential power analysis Annual International Cryptology Conference. Springer, Berlin, Heidelberg, 1999.

[2] Brier, Eric, Christophe Clavier, and Francis Olivier. 2004. Correlation power analysis with a leakage model. International workshop on cryptographic hardware and embedded systems. Springer, Berlin, Heidelberg, 2004.

[3] Suresh Chari, Joshula R. Rao and Pankaj Rohatgi. 2003. Template Attacks. CHES 2002, LNCS, 2523, pp. 13-29, 2003.

[4] H. Maghrebi, T. Portigliatti, and E. Prouff. 2016. Breaking cryptographic implementations using deep learning techniques. In proc. Int. Conf. Security Privacy Appl. Cryptography Eng., 2016, pp. 3-26.

[5] Benjamin Timon. 2018. Non-Profiled Deep Learning-Based Side-Channel Attacks. IACR Cryptol. ePrint Arch., 2018.

[6] Cagli, Eleonora, Cècile Dumas, and Emmanuel Prouff. 2017. Convolutional Neural Networks with Data Augmentation Against Jitter-Based Countermeasures. International Conference on Cryptographic Hardware and Embedded Systems. Springer, Cham, 2017.

[7] S. Hou, Y. Zhou, H. Liu and N. Zhu. 2017. Improved DPA attack on rotating Sboxes masking scheme. 2017 IEEE 9th International Conference on Communication Software and Networks (ICCSN), 2017, pp. 1111-1116, doi: 10.1109/ICCSN.2017.8230283

[8] R. Gilmore, N. Hanley and M. O'Neill. 2015. Neural network based attack on a masked implementation of AES. 2015 IEEE International Symposium on Hardware Oriented Security and Trust (HOST), 2015, pp. 106-111, doi: 10.1109/HST.2015.7140247.

[9] Emmanuel Prouff, Remi Strullu, Ryad Benadjila, Eleonora Cagli, and Cecile Dumas. 2018. Study of Deep Learning Techniques for Side-Channel Analysis and Introduction to ASCAD Database. Cryptology ePrint Archive, Report2018/053, 2018.https://eprint.iacr.org/2018/053.

[10] Timon, B. 2019. Non-Profiled Deep Learning-based Side-Channel attacks with Sensitivity Analysis. IACR Transactions on Cryptographic Hardware and Embedded Systems, 2019(2), pp. 107-131. https://doi.org/10.13154/tches.v2019.i2.107-131

[11] Y. -S. Won, D. -G. Han, D. Jap, S. Bhasin and J. -Y. Park. 2021. Non-Profiled SideChannel Attack Based on Deep Learning Using Picture Trace. in IEEE Access, vol. 9, pp. 22480-22492, 2021, doi: 10.1109/ACCESS.2021.3055833.

[12] A. Alipour, A. Papadimitriou, V. Beroulle, E. Aerabi, and D. Hely. 2020. On the performance of non-profiled differential deep learning attacks against an AES encryption algorithm protected using a correlated noise generation based hiding countermeasure. in Proc. Design, Autom. Test Eur. Conf. Exhib. (DATE), Mar. 2020, pp. 614-617

[13] CW1173 ChipWhisperer-Lite, https://rtfm.newae.com/Capture/ChipWhispererLite/

[14] L. Masure, C. Dumas, and E. Prouff, 2018. Gradient visualization for general characterization in profiling attacks. Cryptology ePrint Archive, Report 2018/1196, 2018, https ://eprint.iacr.org/ 2018/1196.

[15] Lundberg, S., Lee, S.I. 2017. A unified approach to interpreting model predictions. arXiv preprint arXiv:1705.07874 (2017)

[16] DPAContestV4, http://www.dpacontest.org/v4/42 doc.php, August 2015

[17] Mehdi-Laurent Akkar and C. Giraud. 2001. An Implementation of DES and AES, Secure against Some Attacks. In Ç.K. Koç, D. Naccache, and C. Paar, editors, Cryptographic Hardware and Embedded Systems - CHES 2001, volume 2162 of Lecture Notes in Computer Science, pp. 309-318. Springer, 2001.

[18] Emmanuel Prouff and Matthieu Rivain. 2008. A Generic Method for Secure S-Box Implementation. In Sehun Kim, Moti Yung, and Hyung-Woo Lee, editors, WISA volume 4867 of Lecture Notes in Computer Science, pp. 227-244. Springer, 2008.

[19] M. Nassar, Y. Souissi, S. Guilley and J. Danger. 2012. RSM: A small and fast countermeasure for AES, secure against 1st and 2nd-order zero-offset SCAs. 012 Design, Automation \& Test in Europe Conference \& Exhibition (DATE), 2012, pp. 1173-1178, doi: 10.1109/DATE.2012.6176671 


\section{APPENDIX}

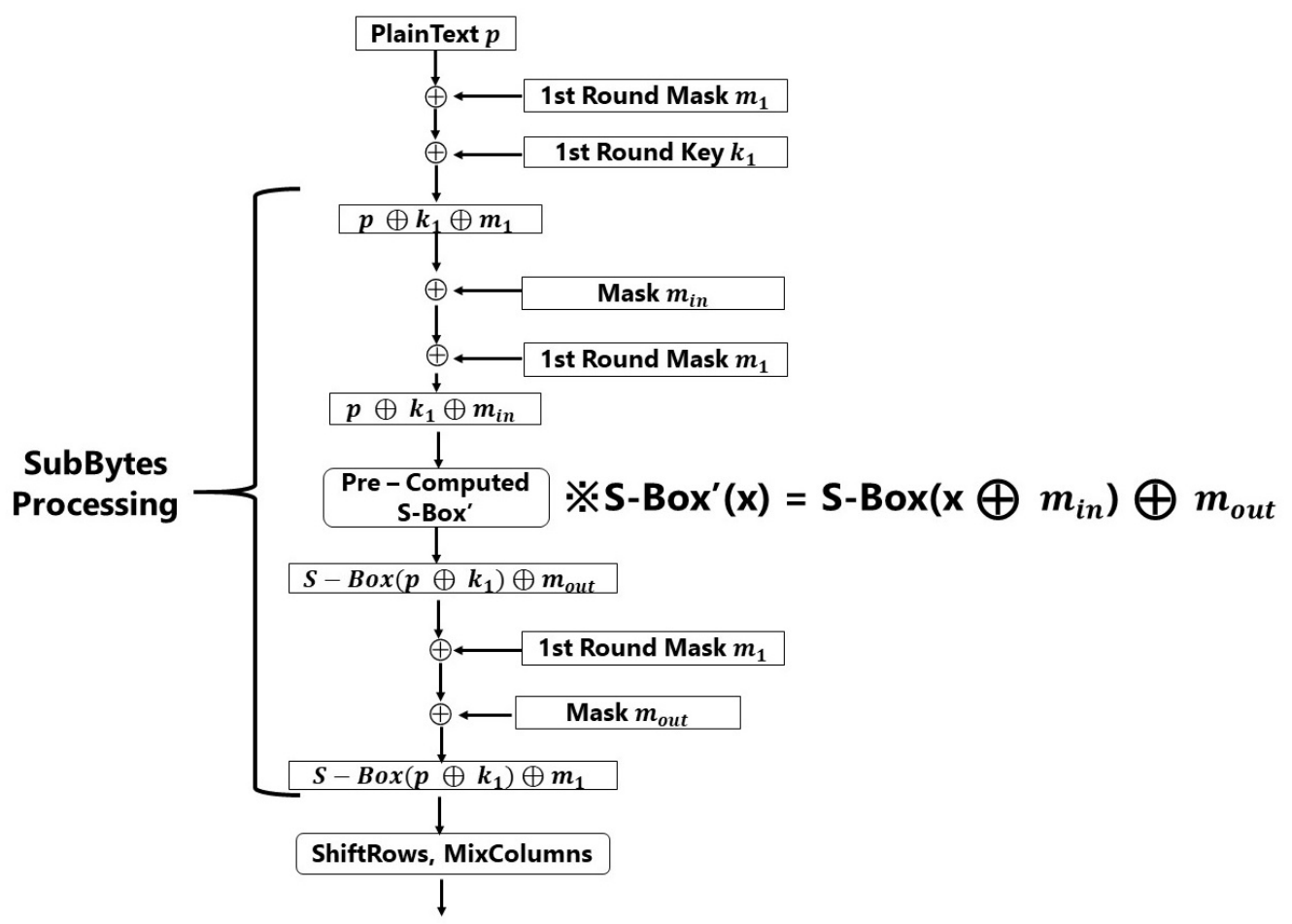

Figure 18: The Overview of 1st Round processing of table re-computation masking AES

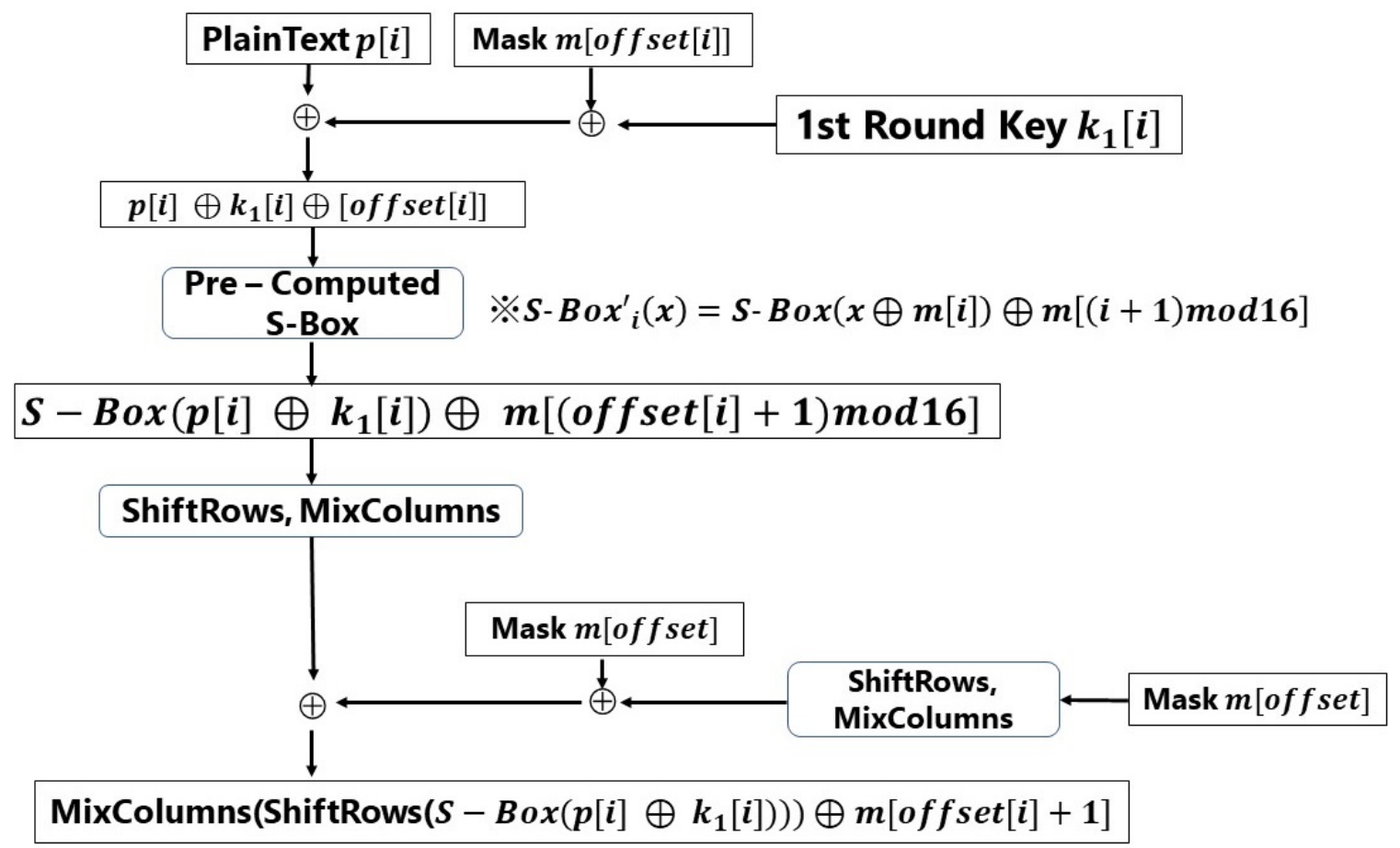

Figure 19: An overview of the i-byte of the first round RSM countermeasure AES 


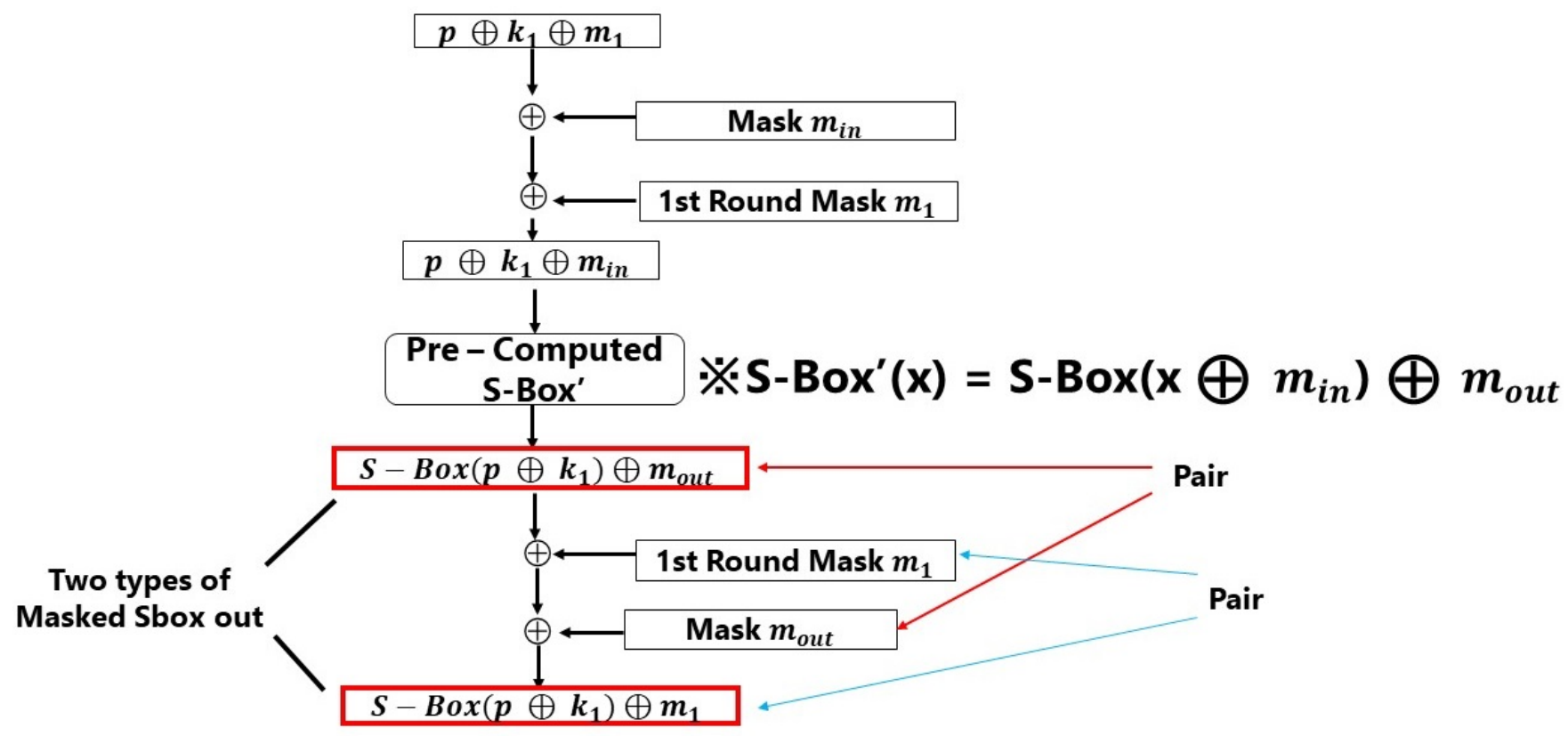

Figure 20: An Overview of the processing corresponding to PoI when attacking against ASCAD

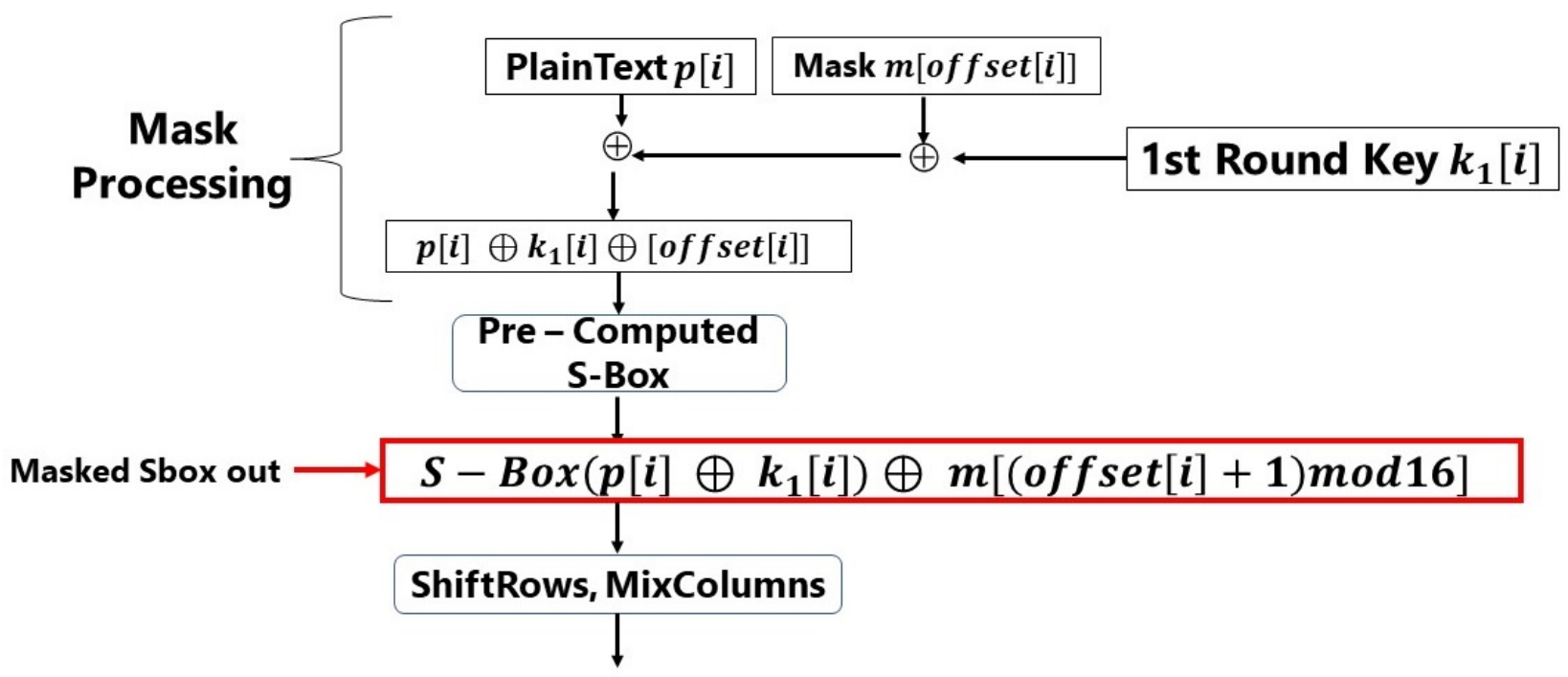

Figure 21: An Overview of the processing corresponding to PoI when attacking against RSM countermeasure AES 